\title{
Solution Electrospinning of Polypropylene-based Fibers and Their Application in Catalysis
}

\author{
Emine Berber, Nesrin Horzum ${ }^{1}$, Baki Hazer², and Mustafa M. Demir ${ }^{3 *}$ \\ Department of Biotechnology and Bioengineering, Faculty of Engineering, Izmir Institute of Technology, İzmir 35430, Turkey \\ ${ }^{1}$ Department of Engineering Sciences, Faculty of Engineering and Architecture, Izmir Katip Çelebi University, \\ İzmir 35620, Turkey \\ ${ }^{2}$ Department of Chemistry, Faculty of Science and Letters, Bülent Ecevit University, Zonguldak 67100, Turkey \\ ${ }^{3}$ Department of Materials Science and Engineering, Faculty of Engineering, Izmir Institute of Technology, Izmir 35430, Turkey
}

(Received January 26, 2016; Revised April 11, 2016; Accepted April 16, 2016)

\begin{abstract}
Since the dissolution of polyolefins is a chronic problem, melt processing has been tacitly accepted as an obligation. In this work, polypropylene (PP) was modified on molecular level incorporating poly(ethylene glycol) (PEG) as graft segment (PP-g-PEG) in a range of 6 to $9 \mathrm{~mol} \%$. Gold nanoparticles were nucleated in the presence of the copolymer chains via redox reaction. The dissolution of the amphiphilic comb-type graft copolymers containing gold nanoparticles ( $80 \mathrm{~nm}$ in diameter) was achieved in toluene and successfully electrospun from its solution. The diameter of composite fibers was in the range from 0.3 to $2.5 \mu \mathrm{m}$. The design of the structurally organized copolymer fiber mats provided a support medium for the nanoparticles enhancing the active surface area for the catalytic applications. The resulting composite fibers exhibited rapid catalytic reduction of methylene blue (MB) dye in the presence of sodium borohydride $\left(\mathrm{NaBH}_{4}\right)$ compared to corresponding composite cast film.
\end{abstract}

Keywords: Catalysis, Comb-type amphiphilic polymer, Electrospinning, Gold nanoparticles, Grafting

\section{Introduction}

Electrospinning is a fiber fabrication process that provides the formation of $\mathrm{km}$ long organic and/or inorganic fibers within submicron/micro meter range diameter with a well-controlled fiber morphology and surface chemistry $[1,2]$. This process is convenient for almost any soluble co/polymer if its molecular weight is high enough. Electrospun fibers have large surface area per unit mass because of their small diameters so that they have high potential for various applications such as drug delivery, catalysis, sensors, functional textiles, tissue engineering, ion exchange membranes, bioengineering etc. [2].

Polypropylene (PP) is high molecular linear addition form of propene [3]. The fibers of PP have high commercial concern because of their good mechanical strength, hydrophobicity, and chemical resistance [4]. Electrospinning is a frequently used technique for the last few decades for the fabrication of fibrous materials, and it was applied successfully to large variety of polymers [5]. However, electrospinning of polyolefin derivatives requires melting therefore the melt needs to be kept at a high temperature. Polyolefins also require fractional dissolution of polymer in nonpolar solvents, which have a low dielectric constant and poor conductivity. Larrondo and Manley firstly applied electrical field to melt form of polyethylene at $200-220^{\circ} \mathrm{C}$. They obtained electrospun PP fibers from a paraffin solution at $100{ }^{\circ} \mathrm{C}[3,6,7]$. The major drawback of this process was the removal of paraffin after electrospinning. By washing the

*Corresponding author: mdemir@iyte.edu.tr fibers in a xylene, the paraffin was removed; however, the fibers were swollen. Givens et al. [8] proposed the electrospinning of low density polyethylene from $p$-xylene solution. The selection of the solvent was appropriate in terms of solvent removal due to its higher volatility but the dielectric constant of $p$-xylene was still low for electrospinning. These limitations were addressed by the addition of salt to the polymer solution and altering the electrospinning set up that allows high temperature process. Electrospinning of polyolefins at slightly elevated temperature was reported by Rabolt et al. [9]. Multicomponent solvent system consisting of cyclohexane, acetone and dimethylformamide was used to prepare syndiotactic polypropylene fibrous membrane. Ultrahigh-molecular-weight polyethylene (UHMWPE) fibers from a mixture of $p$-xylene and cyclohexanone fabricated by Rein et al. [10]. In the fabrication of micro- and nanofibers of polyolefins presented so far, a high temperature environment is required.

Since the difficulty of dissolving polyolefins due to their nonpolar structure, processing at room temperature is not plausible. Therefore, molecular modification appears out to be the only solution to obtain homogeneous dissolution. Reenen et al. reported a simple way to electrospin polyolefins introducing higher 1-alkenes as comonomers, which lowers crystallinity and increases solubility [11].

In this work, amphiphilic graft copolymer containing polypropylene and polyethylene glycol was synthesized and functionalized by gold nanoparticles. We focus on the preparation of PP-based fibers from solution at ambient temperature. Various solvents having different polarity have been used in solubility test of the $\mathrm{PP} / \mathrm{Au}$ composites at 
various electrical field strengths ranging from 1.0 to $1.5 \mathrm{kV} \mathrm{cm}^{-1}$. The catalytic activity of the fibrous system was studied in a model chemical reaction taking place between methylene blue (MB) and sodium borohydride $\left(\mathrm{NaBH}_{4}\right)$.

Most of the organic reagents are not soluble in water as they require some hydrophobic support to catalyze reactions successfully. Electrospun fiber mats are good candidate as support for the metal nanoparticles providing the high catalytic activity and ease of isolation from the reaction system [12]. Palladium particles supported by statistical copolymer of acrylonitrile and acrylic acid were prepared for the hydrogenation reaction of dehydrolinalool (3,7-dimethyloct6-ene-1-yne-3-ol, DHL). The products of this reaction have been used in industrial synthesis of vitamins [13].

Catalytic performance of metal nanocomposites depends on their three important characteristics: i) high surface area of the substrate [14-16], ii) accessibility of the nanoparticle to the reagents [16] and iii) uniformity of the nanoparticles in size and distribution [17]. There are various types of substrates using for the metal nanoparticle encapsulation as metal oxides [14,17], organically modified silicates [18], thin films [19,20], fibers [13], and dendrimers [21]. Considering these substrate types, fiber formed materials display more efficient catalytic performance as compared to the others because of their high surface area, low resistance to flow of gas and liquids, flexible structure, and safer operation conditions [22]. Because of these unique properties of fiber formed materials, they may be used as support for catalytically active species. Particularly, polypropylene fibers have very good solvent resistance that allows the application in various organic media.

\section{Experimental}

\section{Materials}

Chlorinated polypropylene (PP-Cl, Mw 150,000 Da, three repeating units have one chlorine atom on average), polyethylene glycol with different molecular weights (from 400 to $\left.80000 \mathrm{~g} \mathrm{~mol}^{-1}\right)$, sodium hydride $(\mathrm{NaH}$, dispersed in oil $60 \mathrm{wt} \%)$, hydrogen tetrachloroaurate $\left(\mathrm{HAuCl}_{4}\right)$ were supplied from Sigma-Aldrich and used as received. Tetrahydrofuran (THF) was supplied from Sigma-Aldrich and distilled from sodium flakes before use. $N, N$ Dimethylformamide (Carlo Erba, 99\%), toluene (SigmaAldrich, 99.5\%), chloroform (Sigma-Aldrich, $99.4 \%$ ), 1,3dichlorobenzene (Merck, >96\%), methylene blue (AppliChem) were all used as received without any further purification. Demineralized water was used throughout the study.

\section{Synthesis of Gold Nanoparticles Embedded PP-g-PEG Amphiphilic Graft Copolymers}

PP-g-PEG amphiphilic graft copolymers were synthesized by previously reported procedure [23]. Typically, $\mathrm{PP}-\mathrm{Cl}$, $1.5 \mathrm{~g}(10 \mathrm{mmol} \mathrm{Cl})$ was dissolved in $50 \mathrm{~m} l$ of freshly distilled THF. To this solution was added drop wise $20 \mathrm{ml}$ of a THF solution containing PEG2000 (4.0 g, $2 \mathrm{mmol}$ ) and $0.4 \mathrm{~g}$ of $\mathrm{NaH}$. After stirring $3 \mathrm{~h}$ at room temperature, the reaction mixture was poured into $500 \mathrm{ml}$ of methanol containing $1 \mathrm{~m} l$ of concentrated $\mathrm{HCl}$. The polymer was filtered, washed with water and dried under vacuum overnight. For the purification, it was redissolved in THF and reprecipitated in $200 \mathrm{~m} l$ of distilled water and then dried under vacuum overnight at $40{ }^{\circ} \mathrm{C}$.

A series of gold nanoparticles embedded PP-g-PEG amphiphilic graft copolymers were prepared by modifying a previously reported procedure developed [24]. In Table 1, the amounts of the reagents are shown. Aqueous solution of $0.1 \mathrm{M} \mathrm{HAuCl}_{4}$ and the reducing agent, $\mathrm{NaBH}_{4}(0.1 \mathrm{M})$ were prepared separately. The PP-g-PEG graft copolymer $(0.45 \mathrm{~g})$ was dissolved in $15 \mathrm{ml}$ of THF. To this solution 0.02 or $0.06 \mathrm{ml}$ of the $\mathrm{HAuCl}_{4}$ aqueous solution was added and vigorously stirred at room temperature for $10 \mathrm{~min}$. Then, the

Table 1. Ingredients for the preparation of gold nanoparticles embedded PP-g-PEG amphiphilic comb-type graft copolymers

\begin{tabular}{|c|c|c|c|c|c|c|c|}
\hline Polymer code & $\mathrm{PP}-\mathrm{Cl}(\mathrm{g})$ & PEG type (g) & $\mathrm{NaH}(\mathrm{g})$ & Yield (g) & Water uptake (wt $\%$ ) & PEG (mol\%) & AuNPs (mg) \\
\hline PPEG 400-1 & 5.1 & PEG400/7.4 & 1.87 & 5.8 & 14 & 18 & 0.7 \\
\hline PPEG 1000-1 & 6.0 & PEG1000/4.2 & 1.13 & 6.8 & 26 & 17 & 2.0 \\
\hline PPEG 1000-2 & 6.0 & PEG1000/12.8 & 1.24 & 7.4 & 26 & 18 & 2.0 \\
\hline PPEG 2000-1 & 6.4 & PEG2000/10 & 0.70 & 6.8 & 46 & 22 & 0.7 \\
\hline PPEG 2000-2 & 5.2 & PEG2000/7.5 & 1.84 & 5.3 & 12 & 21 & 2.0 \\
\hline PPEG 3350-1 & 1.3 & PEG3350/0.72 & 0.18 & 1.3 & 28 & 9 & - \\
\hline PPEG 3350-2 & 1.3 & PEG3350/0.72 & 0.18 & 1.3 & 28 & 9 & 2.0 \\
\hline PPEG 3350-3 & 1.37 & PEG3350/5.2 & 0.18 & 1.48 & 59 & 18 & 2.0 \\
\hline PPEG 3350-4 & 1.30 & PEG3350/1.32 & 0.18 & 1.25 & 28 & 11 & 2.0 \\
\hline PPEG 3350-5 & 8.7 & PEG 3350/4.0 & 0.27 & 10.7 & 15 & 15 & 2.0 \\
\hline PPEG 4000 & 5.3 & PEG4000/8.1 & 0.78 & 4.1 & 17 & 19 & 2.0 \\
\hline PPEG 8000-1 & 4.0 & PEG8000/0.53 & 0.34 & 5.9 & 12 & 11 & 2.0 \\
\hline PPEG 8000-2 & 6.1 & PEG8000/2.3 & 1.70 & 6.7 & 13 & 17 & 2.0 \\
\hline PPEG 80000 & 5.4 & PEG80000/9.0 & 0.48 & 4.9 & 30 & 19 & 2.0 \\
\hline
\end{tabular}


equivolume of the reducing agent of $\mathrm{NaBH}_{4}$ aqueous solution was added to this mixture, generating a purple color colloidal solution. The solution was filtered and poured into a Petri dish, and the solvent was allowed to evaporate leaving a purple colored thin polymer film. The solvent cast film was washed with methanol and dried under vacuum at $40{ }^{\circ} \mathrm{C}$ for $24 \mathrm{~h}$.

\section{Electrospinning Process}

PP-g-PEG copolymer films containing different amount of gold nanoparticles were attempted to dissolve in $N, N$ Dimethylformamide, toluene, chloroform, or dichlorobenzene. PPEG 4000, PPEG 3350-4, PPEG 3350-5, PPEG 8000-1, and PPEG 8000-2 were investigated for their electrospinning characteristics. PPEG 3350-4 was dissolved in $5 \mathrm{wt} \%$ toluene and PPEG 4000, PPEG 3350-5, PPEG 8000-1, PPEG 8000-2 were dissolved in $10 \mathrm{wt} \%$ toluene. The prepared polymer solutions were supplied into a $20 \mathrm{~m} l$ syringe with a metal capillary needle (inner diameter $=0.8 \mathrm{~mm}$ ). The electrospinning process was performed at room temperature and the solutions were injected from the syringe pump (LION WZ-50C6) with a feed rate of $1.5 \mathrm{~m} / \mathrm{h}^{-1}$. Using a high-voltage power supply (Gamma High Voltage Research Ormond Beach, FL, US) at a range of 20 to $30 \mathrm{kV}$, a jet was ejected from the needle tip. The electrospun nanofibers were assembled on a stable collector (coated with aluminum foil) placed at $20 \mathrm{~cm}$ distance.

\section{Characterization Methods}

The surface morphology and diameter of the electrospun nanofibers and polymer films were observed by a scanning electron microscope (SEM, FEI Quanta250 FEG, Oregon, USA). For quantification of average fiber diameters and diameter of the gold nanoparticles, the fibers/particles were randomly selected from each SEM micrographs and the diameters of each were measured using Image-J software. Thermogravimetric analysis (TGA) was studied by a Perkin Elmer Diamond TG/DTA. The molecular weight of the copolymers was determined by gel permeation chromatography (GPC) measurements in tetrahydrofuran (THF) with an Agilent 1100 Series GPC Setup including a Zorbax PSM 60 S column (range: 5×102-104 MW), Zorbax PSM $1000 \mathrm{~S}$ (range: 104-106 MW), UV (254 nm), and refractive index (RI) detector. The eluent was run at $40{ }^{\circ} \mathrm{C}$ with the flow rate of $1 \mathrm{~m} l \mathrm{~min}^{-1}$. A calibration curve was generated with four polystyrene green standards provided by EasyCal Agilent Technologies Polymer Standards Service (MW's: 696500, 50400, and 2960).

The catalytic activity of the gold nanoparticles embedded PP-g-PEG copolymer was examined employing a model redox reaction of methylene blue $(\mathrm{MB})$ and sodium borohyride $\left(\mathrm{NaBH}_{4}\right)$. For the reduction of $\mathrm{MB}$, a mixture containing aqueous solution of methylene blue $(3 \mathrm{ml}, 0.02 \mathrm{mM})$ and $\mathrm{NaBH}_{4}(1 \mathrm{ml}, 0.1 \mathrm{mM})$ was prepared in a quartz cuvette.
Then $0.20 \mathrm{mg}$ of PPEG 3350-5 copolymer film was added to the solution. For comparison, an electrospun mat of PPEG $3350-5$ copolymer $(0.20 \mathrm{mg})$ was immersed into the mixture of $3 \mathrm{~m} l$ of $0.02 \mathrm{mM}$ methylene blue and $1 \mathrm{~m} l$ of $0.1 \mathrm{mM}$ $\mathrm{NaBH}_{4}$. A UV-visible spectrometer (UV SHIMADZU 2450, Japan) was used to monitor their reaction kinetics by measuring their time-dependent absorption spectra.

\section{Results and Discussion}

PP-g-PEG amphiphilic copolymers are water swellable, elastic and biocompatible materials [25,26]. They are suitable to prepare nanocomposite containing metal nano particles or quantum dots [27]. PP-g-PEG copolymer containing gold or cobalt oxide nanoparticles was successfully used in an enzymatic fuel cell for renewable fuels [28]. Gold nanoparticle embedded PP-g-PEG amphiphilic copolymer fibers presented in this work were obtained via electrospinning. Micro sized fiber mat formation provided a supporting medium for the gold nanoparticles and electrospun copolymer fibers showed enhanced catalytic reduction of methylene blue (MB) dye in the presence of sodium borohydride $\left(\mathrm{NaBH}_{4}\right)$ in comparison with the copolymer film.

\section{Synthesis of Gold Nanoparticles Embedded PP-g-PEG Amphiphilic Graft Copolymers}

Scheme 1 shows step-by-step evolution of PP-g-PEG amphiphilic comb-type graft copolymers. First, chlorinated$\mathrm{PP}$ is treated with base in organic medium and PEG molecules are grafted onto the backbone of the PP. Second, $\mathrm{Au}$ precursors are reacted with PEG of the resulting amphiphilic copolymer chains.

Figure 1(a), 1(b), and 1(c) show representative overview SEM images of the cast films containing 11,17 , and $15 \%$ of PEG, respectively. The amount of Au particles is fixed to $2 \mathrm{mg}$ in all samples. The back-scattered electron detector showed that the variation in brightness over the surface represents the localized separation of PP and PEG segments. Three phases exist in the images. The dark background is PP

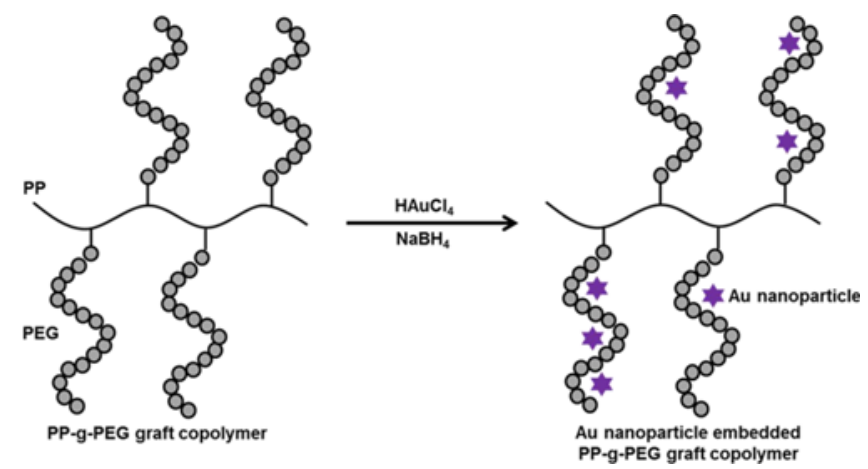

Scheme 1. Synthesis steps for gold nanoparticle embedded PP-gPEG graft copolymer. 


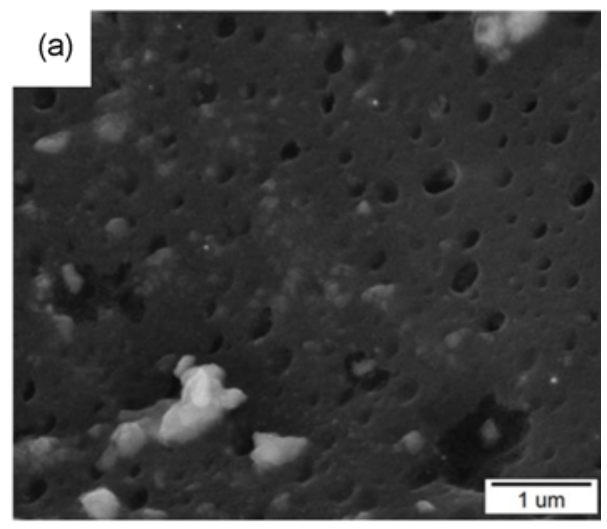

(b)
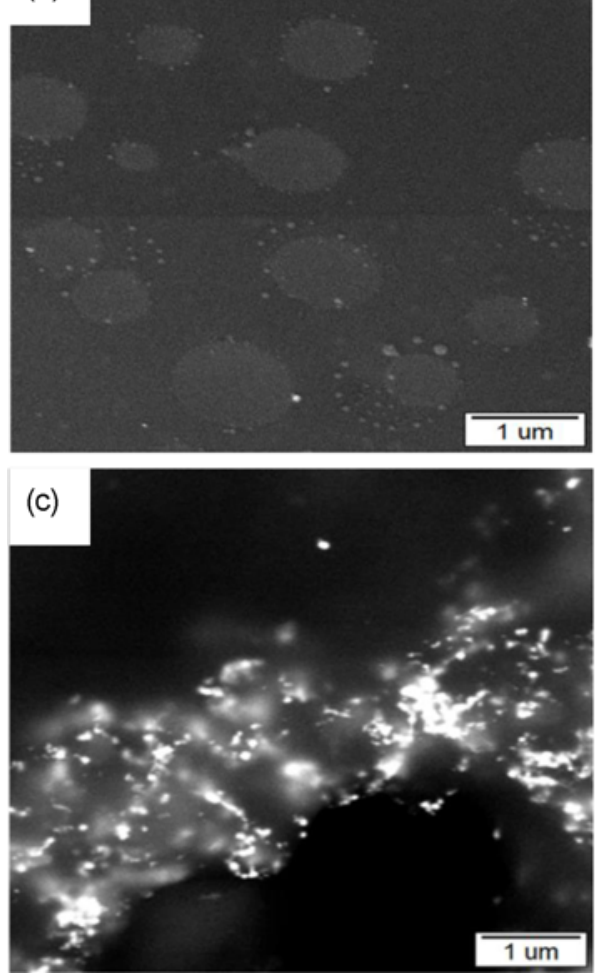

Figure 1. SEM micrographs of the synthesized copolymer species (a) PPEG 8000-1, (b) PPEG 8000-2, and (c) PPEG 3350-5.

matrix. The bright phases refer to Au particles. On the other hand, gray regions can be attributed to chlorinated-PEG domains. Since atomic number of $\mathrm{Au}$ is higher than matrix atoms of carbon and chlorine, Au particle domains strongly scatter electron beam. In addition, energy dispersive X-ray analysis (EDX) of the gold nanoparticles embedded graft copolymer sample is shown in Figure 2. The size of bright Au domains is $74 \mathrm{~nm}$. The domains may include single and/ or a group of individual particles. In addition to Au domains, larger and grey spherical domains are evident in the microscopy image. Characteristic X-ray emission of the chlorine element is examined for different location of the samples for instance inside and outside of the spherical

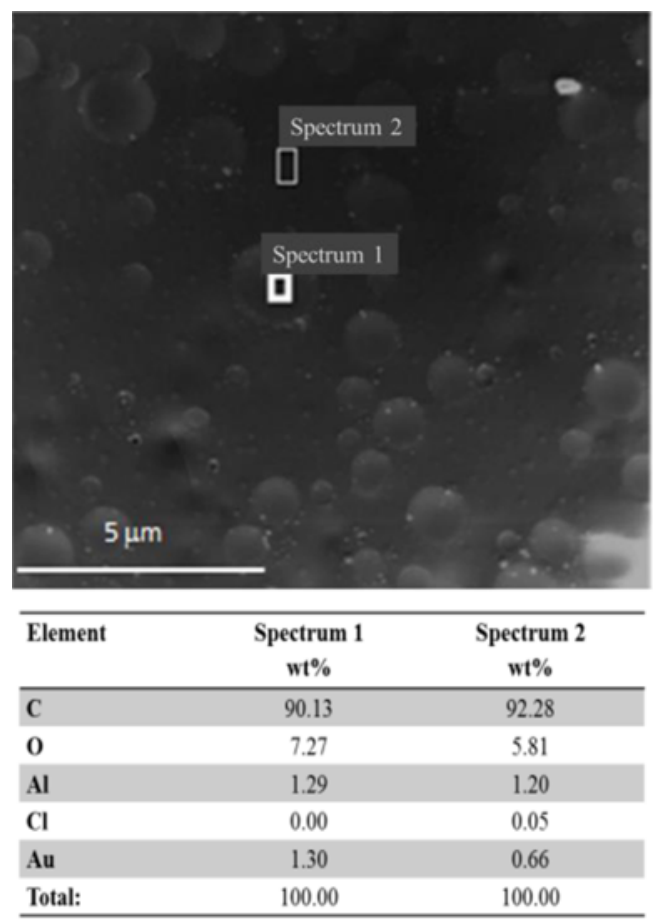

Figure 2. EDX analysis of the indicated area of the copolymer PPEG 8000-2.

domains. The comparison between the elemental compositions of these phases in the matrix suggests that the domains are almost free of chlorine molecules (Spectrum 1). However, the matrix consists of nearly $0.05 \%$ chlorine atoms by mass (Spectrum 2).

The origin of this phase separation could be the molecules that have been successfully modified with PEG. Due to thermodynamic incompatibility between chlorine rich and lean chains, a microphase separation may occur. Note that $\mathrm{Au}$ nanoparticles seem preferentially locate chlorine lean chains outside of the spherical domains. Such selective loading has been observed in composites prepared by copolymers [29] or polymer blends [30].

Thermal behavior of the PPEG 400-1, PPEG 2000-1, and PPEG 3350-5 films and their relative gold content was determined by TGA. Figure 3 presents the thermograms of the samples. According to TGA data, two consecutive mass losses were observed. They can be attributed to the elimination of PEG segments and chlorinated PP segments, respectively [23]. TGA curve of PPEG 400-1 and PPEG 2000-1 were almost identical. The remaining mass at $550{ }^{\circ} \mathrm{C}$ for PPEG 3350-5 film was higher (64 \%) than PPEG 400-1 and PPEG 2000-1 films (7\%). The thermal stability of the fibers increased upon the increase of gold content. The onset of degradation temperature of PEG segment shifted about $100^{\circ} \mathrm{C}$ in the presence of gold nanoparticles.

Saldias et al. [31] reported that gold or silver nanoparticlecopolymer system using poly(ethylene glycol)-poly( $\varepsilon$ - 


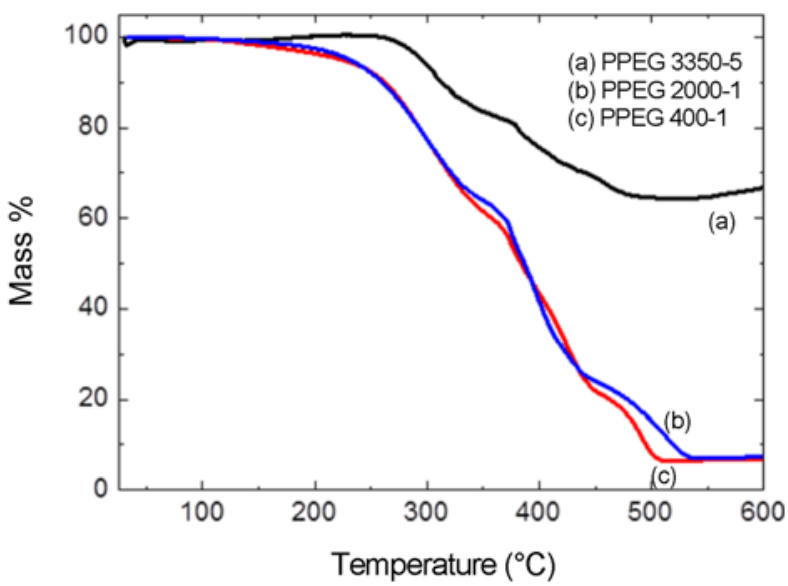

Figure 3. Thermogravimetric analysis (TGA) curves for the copolymer species, PPEG 400-1, PPEG 2000-1, and PPEG 3350-5.

caprolactone) (PEG-PCL) block copolymers hindered the decomposition of the neat PEG-PCL. Since the degradation mechanism relies on the diffusion of polymer residues formed upon increase of temperature and the corresponding change in the molecular mobility of the polymer chains, the nanoparticles may act as barrier for mass transport leading to the improvement of the thermal properties of the materials.

\section{Electrospinning Process and Morphology of Electrospun Nanofibers}

Table 2 summarizes the list of the copolymers synthesized, their solubility in different solvents, and the electrospinnability of the solution. Although the composition of the chains is not very much different, some of the polymers are successfully electrospun, whereas some of them are not. Three processing variables, solution flow-rate, applied voltage, and tip-tocollector distance were studied for the all species. Fibers (PEG 3350-4, PPEG 3350-5, PPEG 4000, PPEG 8000-1, and PPEG 8000-2) were electrospun from the solutions of $5 \mathrm{wt} \%$ and $10 \mathrm{wt} \%$ copolymers in toluene. These samples successfully allow the fabrication of continuous and stable electrospun fibers. Note that for such complex systems containing many material components, the process strongly based on the precursor solution properties. Concentration of the solution/dispersion seems the dominant parameter. Electrospinning can be successfully applied when the polymer concentration exceeds $10 \mathrm{wt} \%$ in solution. For lower concentration, the electrical potential applied to the polymer solution atomizes the solution/dispersion and small droplets occur. Electrospinning shifts to electrospraying regime. Moreover, complete dissolution was not achieved in dichloromethane.

Electrospinning of polypropylene is problematic since the complete dissolution of polyolefin cannot be achieved in any solvents at room temperature. Structurally consistent electrospun fibers were fabricated from poly(ethylene glycol)/gold
Table 2. Solubility and electrospinnability characteristics of the synthesized PP-g-PEG amphiphilic comb-type graft copolymers (voltage: $25.5 \mathrm{kV}$, distance: $20 \mathrm{~cm}$, and feed rate: $1.5 \mathrm{~m} l \mathrm{~h}^{-1}$ )

\begin{tabular}{llcc}
\hline \multicolumn{1}{c}{ Polymer } & \multicolumn{1}{c}{ State of dissolution } & $\begin{array}{c}\text { Solid } \\
\text { content } \\
(\mathrm{wt} \%)\end{array}$ & $\begin{array}{c}\text { Electro- } \\
\text { spinnability }\end{array}$ \\
\hline PPEG 400-1 & Soluble in dichlorobenzene & 5 & No \\
PPEG 1000-1 & Soluble in dichlorobenzene & 5 & No \\
PPEG 1000-2 & Partial dissolution in chloroform & 5 & No \\
PPEG 2000-1 & Partial dissolution in toluene, & 5 & No \\
& Soluble in dichlorobenzene & & \\
PPEG 2000-2 & Soluble in toluene & 5 & No \\
PPEG 3350-2 & Soluble in toluene & 2 & No \\
PPEG 3350-3 & Soluble in toluene & 5 & Yes \\
PPEG 3350-4 & Soluble in toluene & 10 & Yes \\
PPEG 3350-5 & Soluble in toluene & 10 & Yes \\
PPEG 4000 & Soluble in toluene & 10 & Yes \\
PPEG 8000-1 & Soluble in toluene & 10 & Yes \\
PPEG 8000-2 & Soluble in toluene & 5 & No \\
PPEG 80000 & Soluble in toluene & &
\end{tabular}

nanoparticle modified polypropylene using the polymer solution (10 $\mathrm{wt} \%$ in toluene). Figure 4 shows the SEM micrographs of the gold nanoparticle modified PP-g-PEG electrospun fibers and the corresponding fiber diameter distributions. The composite electrospun fibers have a smooth surface without any bead formation. The mean diameter of the fibers shows variation ranging between 0.3 to $2.5 \mu \mathrm{m}$ depending on the polymer content and solvent characteristics.

Among the diameter distributions for the species PPEG 8000-1, PPEG 8000-2, and PPEG 3350-5, the higher average fiber diameter $(\sim 2 \mu \mathrm{m})$ was obtained for PPEG $3350-5$. The increment may be attributed to the higher gold content of the copolymer. In addition, increasing conductivity decreases the flow resistance of the polymer solution so that the higher feed rate requirement results in the formation of thicker fibers [32].

Figure 5 presents the representative SEM micrographs of PPEG 3350-3 copolymer film and electrospun PPEG 8000-1 fiber. The size of the gold nanoparticles in copolymer films and fibers were identified from the back scattered electrons detection mode of the SEM. While gold nanoparticles on the copolymer film are with a diameter of $75 \mathrm{~nm}( \pm 15)$, those on the fibers have diameters ranging from 150 to $250 \mathrm{~nm}$. The particle domains are larger in electrospun fiber system. They may be charged negatively in the polymer/particle dispersion. De-agglomeration of the particles may be expected. However, when the spinning jet arrives the surface of counter grounded electrode (collector), they are neutralized such that they diffuse each other and may form large agglomerates during the solidification of the electrospun fibers. The main 

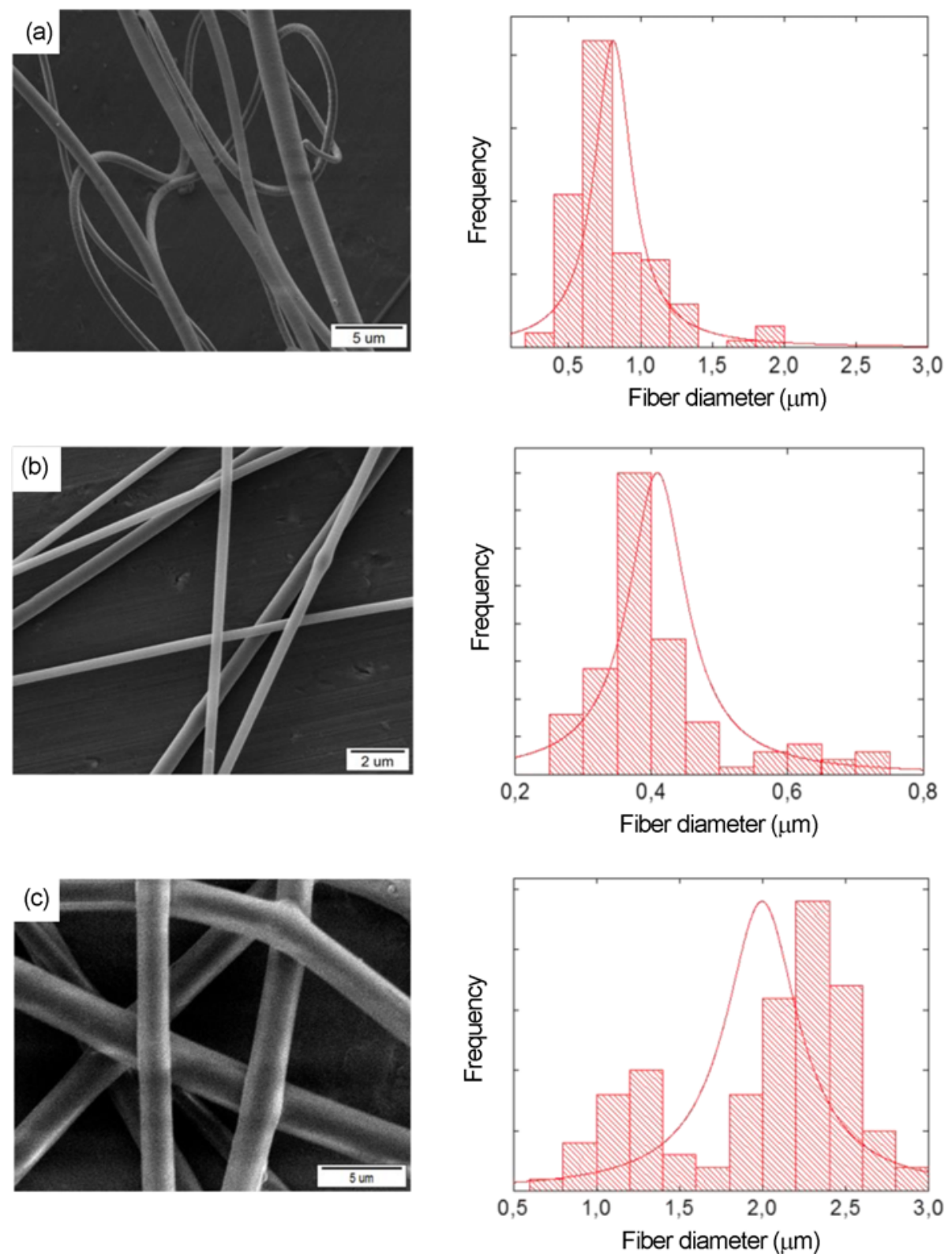

Figure 4. SEM micrographs and diameter analysis of electrospun (a) PPEG 8000-1, (b) PPEG 8000-2, and (c) PPEG 3350-5 fibers from $10 \mathrm{wt} \%$ of the polymer in toluene solutions.

question in this particular system is to control Au particles domain size as a function of PEG content in PP-PEG. However, a solid conclusion was not found depending on our results, at least in the experimental conditions employed in this study.

\section{Catalytic Activity of the PP-g-PEG/Au Composites}

The catalytic activity of the gold nanoparticle modified copolymer is tested in a model reaction taking place between methylene blue dye and a reducing agent of $\mathrm{NaBH}_{4}$. The results of cast and electrospun film are presented in Figure 6. The extent of the reaction was determined by following the characteristic absorption band of MB at $665 \mathrm{~nm}$.

As shown in Figure 6(a), the reduction of MB is not possible in the presence of $\mathrm{NaBH}_{4}$ under ambient conditions unless there is a strong catalyst in the reaction medium [33]. In the presence of $0.20 \mathrm{mg}$ PPEG 3350-5 films, no change in the optical density of the solution during $6 \mathrm{~min}$ refers that the extent of the occurred reduction is lower than the detection limit of optical spectroscopy (Figure 6(b)).

On the other hand, in the solution containing PPEG 3350-5 fibers, the reduction of the dye by $\mathrm{NaBH}_{4}$ is almost completed within 2 min. (Figure 6(c)). It was observed that for a given exposure time, the fibrous sample showed a significant 

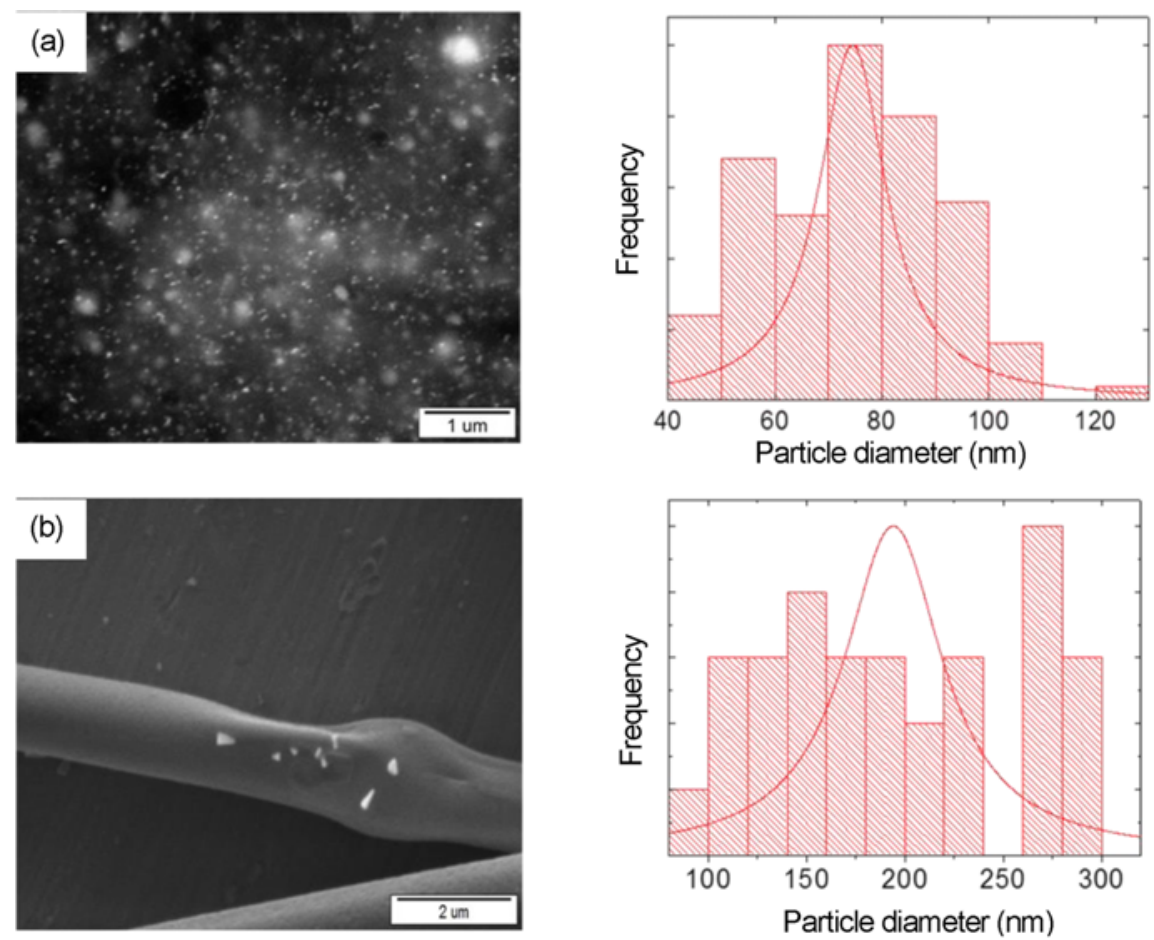

Figure 5. SEM micrograph and the corresponding gold nanoparticle distribution of (a) PPEG 3350-3 copolymer film and (b) electrospun PPEG 8000-1 fibers.
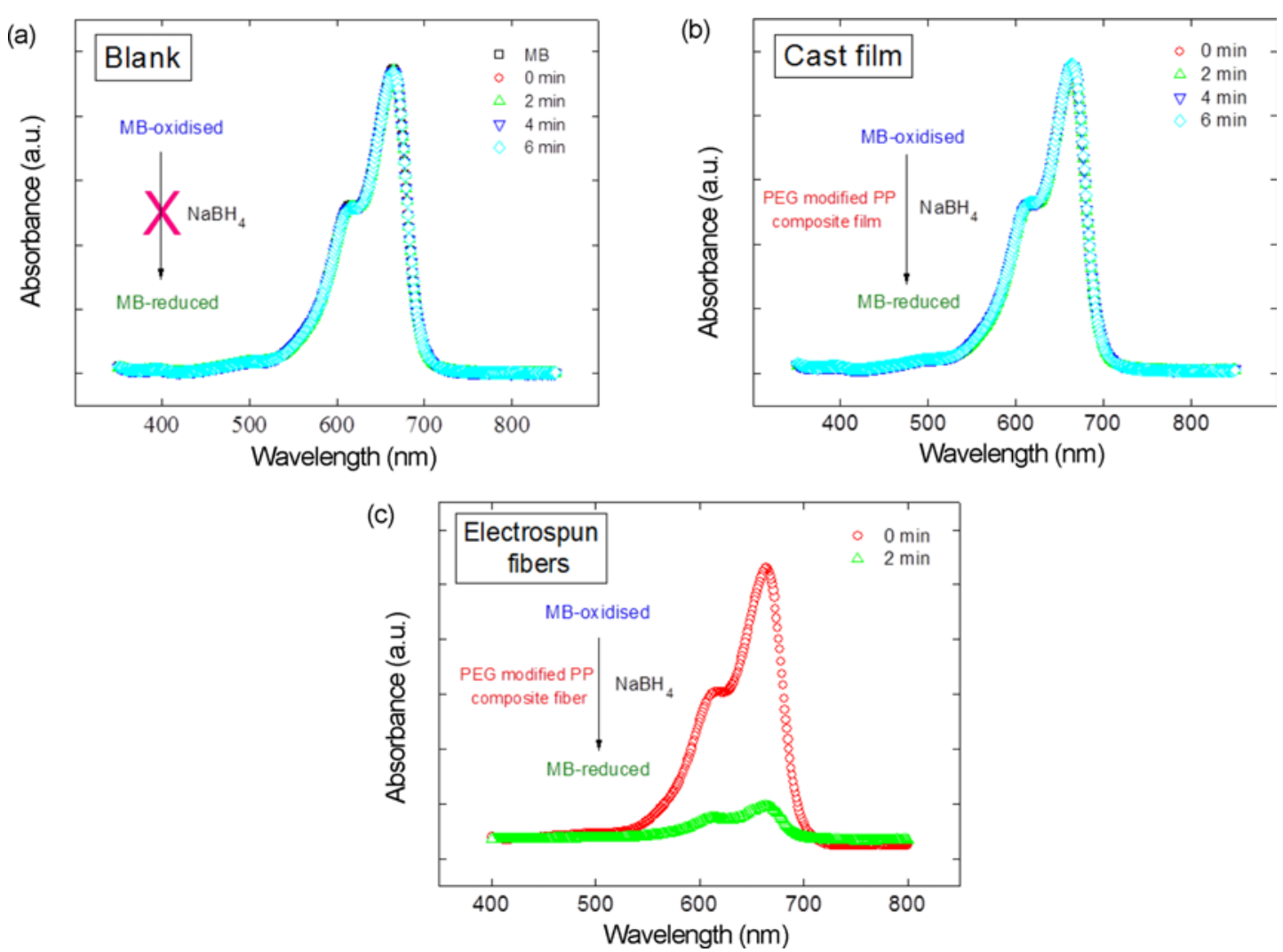

Figure 6. Time-dependent visible spectra of (a) a mixture of $\mathrm{MB}$ dye and reducing agent $\mathrm{NaBH}_{4}$, (b) the mixture in the presence of PPEG 3350-5 film, and (c) the mixture in the presence of electrospun PPEG 3350-5 fibers. 
catalytic activity as compared to its cast film. The reason for the increase in the presence of fibers could be the larger available surface area of the electrospun film compared to cast one. In cast films, the particles are placed both on the film surface and in the film body. Obviously, only the ones placed on the surface have interaction with the surrounding, i.e., contribute to the catalytic activity. However, the particles embedded in the bulk film have no or minimum activity since their surface is already covered by the polymer chains. On the other hand, electrospun fiber mats provide larger surface area. Although the particles size is larger in electrospun fiber system, the number of available particles for the interaction with surrounding (reactants) is higher. As a result, better catalytic activity is achieved.

\section{Conclusion}

Gold nanoparticles embedded PP-g-PEG amphiphilic graft copolymers with different compositions of the PP and PEG were successfully synthesized. Electrospinning of PPbased amphiphilic derivatives from organic solution was performed at room temperature. The catalytic activity of the gold nanoparticle modified PP-g-PEG fibers was validated in a model redox reaction taking place between $\mathrm{NaBH}_{4}$ and $\mathrm{Au}$ salt. The PP-g-PEG fiber mats provide an insoluble polymeric support for catalytically active gold nanoparticles. The catalytic activity of the fiber-supported Au particles is higher than those supported by cast films although they contain identical amount of the Au nanoparticles. The reason may be due to the higher number of available Au nanoparticles present on large surface area of the electrospun fibers compared to cast films. These organic-inorganic hybrid polymers can be potentially applied as support material for catalytic species in industrial reactions taking place in organic medium. This strategy for the development of PP-gPEG fiber supported catalyst can readily be extended to other metal particle systems for the controlled nanostructure formation of polyolefins.

\section{Acknowledgments}

The authors thank the Centre for Materials Research Centre of IZTECH. Partial financial support for this research was provided by the Scientific Research Project BEU-20147211-8496-02.

Electronic Supplementary Material (ESM) The online version of this article (doi: 10.1007/s12221-016-6183-7) contains supplementary material, which is available to authorized users.

\section{References}

1. D. H. Reneker and I. Chun, Nanotechnol., 7, 216 (1996).
2. S. Agarwal, J. H. Wendorff, and A. Greiner, Macromol. Rapid Commun., 31, 1317 (2010).

3. L. Larrondo and R. S. J. Manley, J. Polym. Sci. Pt. BPolym. Phys., 19, 909 (1981).

4. A. Noumowe, Cem. Concr. Res., 35, 2192 (2005).

5. J. Doshi and D. H. Reneker, J. Electrost., 35, 151 (1995).

6. L. Larrondo and R. S. J. Manley, J. Polym. Sci. Pt. BPolym. Phys., 19, 921 (1981).

7. L. Larrondo and R. S. J. Manley, J. Polym. Sci. Pt. BPolym. Phys., 19, 933 (1981).

8. S. R. Givens, Macromol., 40, 608 (2007).

9. K. H. Lee, Macromol., 42, 5215 (2009).

10. D. M. Rein, J. Polym. Sci. Pt. B-Polym. Phys., 45, 766 (2007).

11. A. J. van Reenen and L. Keulder, Macromol. Mater. Eng., 295, 666 (2010).

12. N. Horzum, R. Munoz-Espi, G. Glasser, M. M. Demir, K. Landfester, and D. Crespy, ACS Appl. Mater. Interfaces, $\mathbf{4}$, 6338 (2012).

13. M. M. Demir, M. A. Gulgun, Y. Z. Menceloglu, B. Erman, S. S. Abramchuk, E. E. Makhaeva, A. R. Khokhlov, V. G. Matveeva, and M. G. Sulman, Macromol., 37, 1787 (2004).

14. D. Khushalani, S. Hasenzahl, and S. Mann, J. Nanosci. Nanotechnol., 1, 129 (2001).

15. J. Y. Ying, C. P. Mehnert, and M. S. Wong, Angew. Chem., Int. Ed., 38, 56 (1999).

16. L. M. Bronstein, S. Polarz, B. Smarsly, and M. Antonietti, Adv. Mater, 13, 1333 (2001).

17. M. Epifani, C. Giannini, L. Tapfer, and L. Vasanelli, J. Am. Ceram Soc., 83, 2385 (2000).

18. K. Chakrabarti and C. M. Whang, Mater. Sci. Eng., 88, 26 (2002).

19. M. P. Zheng, M. Y. Gu, Y. P. Jin, and G. L. Jin, Mater. Res. Bull., 36, 853 (2001).

20. S. Bharathi, M. Nogami, and S. Ikeda, Langmuir, 17, 7468 (2001).

21. L. Y. Li, X. B. Cao, F. Yu, Z. Y. Yao, and Y. Xie, J. Colloid Interface Sci., 261, 366 (2003).

22. Y. Matatov-Meytal and M. Sheintuch, Appl. Catal. A-Gen., 231, 1 (2002).

23. M. Balci, A. Alli, B. Hazer, O. Guven, K. Cavicchi, and M. Cakmak, Polym. Bull., 64, 691 (2010).

24. O. A. Kalayci, F. B. Coemert, B. Hazer, T. Atalay, K. A. Cavicchi, and M. Cakmak, Polym. Bull., 65, 215 (2010).

25. D. B. Hazer, B. Hazer, and N. Dincer, Biomed. Biotechnol., 2011, 1 (2011).

26. D. B. Hazer, M. Mut, N. Dincer, Z. Saribas, B. Hazer, and T. Ozgen, Childs Nerv. Syst., 28, 839 (2012).

27. O. A. Kalayci, O. Duygulu, and B. Hazer, J. Nanopart. Res., 15, 1 (2013).

28. M. S. Kilic, S. Korkut, B. Hazer, and E. Erhan, Biosens. Bioelectron., 61, 500 (2014).

29. M. R. Bockstaller, R. A. Mickiewicz, and E. L. Thomas, Adv. Mater., 17, 1331 (2005). 
30. S. Tuzuner and M. M. Demir, Mater. Chem. Phys., 162, 692 (2015).

31. C. Saldías, Á. Leiva, S. Bonardd, C. Quezada, S. Saldías, M. Pino, and D. Radic, React. Funct. Polym., 96, 78
(2015).

32. A. M. El-Rafei, Ceram. Int. B., 41, 12065 (2015).

33. M. M. Demir, G. Ugur, M. A. Gulgun, and Y. Z. Menceloglu, Macromol. Chem. Phys., 209, 508 (2008). 